\title{
Ein Blick in Hossers Küche. Analyse der Rechnungslegung des Küchenmeisters Jakob Hosser über die bei der Belage- rung der Burg Weineck (1292) aufgewendeten Lebensmittel
}

\section{Mattia Pellegrini}

Kerngebiet: Mittelalter

eingereicht bei: ao. Univ.-Prof. Mag. Dr. Julia Hörmann-Thurn und Taxis

eingereicht im: SoSe 2020

Rubrik: Proseminar-Arbeit

\begin{abstract}
A look into Hosser's kitchen. Analysis of chef Jakob Hosser's accounts on food used during the siege of Weineck Castle (1292)

The following seminar paper examines the catering of Otto von Königsberg's troops at the siege of Weineck Castle in 1292. Firstly, the dispute between Meinhard II. and the Bishops of Trient as well as older Tyrolean accounting books are discussed. Then, the primary sources - Hosser's accounts - are analyzed. It will be shown that Hosser's records allow interesting conclusions on the diet of the siege troops and the chef's purchasing of goods. The analysis shows, among other things, that the food supply was highly differentiated, consisted largely of animal products and was subject to certain fluctuations.
\end{abstract}

\section{Einleitung}

Meinhard II. (um 1239-1295), Graf von Tirol-Görz und Herzog von Kärnten, zählt bis heute zu den meistdiskutierten Herrscherpersönlichkeiten im Tiroler Raum. Der Trend zu wissenschaftlichen Publikationen über den „Begründer des Landes Tirol“ und Stammvater der Linie Tirol-Görz ist bis dato ungebrochen.' Trotzdem sind einige Aspekte seiner Person und seiner Taten weitestgehend unerforscht. Die vorliegende Ar-

Zahlreiche Beiträge zum Leben und Wirken Meinhards II. haben Autorinnen und Autoren wie Josef Riedmann, Christoph Haidacher, Franz-Heinz Hye oder Julia Hörmann verfasst. 
beit soll einen Beitrag dazu leisten, einen dieser Aspekte der Geschichte Meinhards II. und damit auch des Landes Tirols greifbar zu machen: Bis zum heutigen Tag gibt die Belagerung der Burg Weineck am Virgl bei Bozen, die im direkten Zusammenhang mit dem Konflikt zwischen Meinhard II. und den Bischöfen von Trient steht, Rätsel auf. Leider sind wenige Zeugnisse über dieses Ereignis überliefert und die langfristige Strategie Meinhards II. nach der Eroberung der Burg bleibt unklar.2 Umso bedeutender scheinen jene wenigen Quellen, die von diesem Ereignis berichten. Allen voran die Rechnungslegungen über die im Zuge der Belagerung aufgewendeten Lebensmittel aus den Tiroler Rechnungsbüchern. Am aufschlussreichsten scheint hierbei die Rechnungslegung des Küchenmeisters Jakob Hosser. Die umfangreichste der drei erhaltenen Dokumentationen über die Versorgung der Belagerer beinhaltet Informationen, die Rückschlüsse über die Vorgänge bei Weineck zulassen.

Die vorliegende Arbeit verfolgt das Ziel, die Approvisation der Belagerungstruppen zu durchleuchten. Es wird der Frage nachgegangen, wie sich die Verpflegung der Belagerer der Burg Weineck gestaltete.

An dieser Stelle soll von keiner allzu abwechslungsreichen Kost ausgegangen werden. Es ist anzunehmen, dass in dieser Kriegssituation weit andere Prioritäten gesetzt wurden, als den Mannschaften Gaumenfreuden zu bereiten. Trotzdem kann von einer gut durchdachten Beschaffung und Organisation der Ressourcen ausgegangen werden, die bei einer erfolgreichen Belagerung unerlässlich sind. Daher soll folgende These überprüft werden: Die Verpflegung der Belagerungstruppen der Burg Weineck war einfach und bescheiden, aber gut geplant und organisiert.

Zu Beginn wird die Rechnungslegung kontextualisiert, bevor die Konfrontation Meinhards II. mit den Bischöfen von Trient und die damit verbundene Rolle der Burg Weineck ins Zentrum der Betrachtungen rückt. Auch wird der Entstehungskontext der Tiroler Rechnungsbücher thematisiert, um anschließend die Approvisation präsentieren und analysieren zu können. Das Ziel der vorliegenden Arbeit ist es nicht, sämtliche Unklarheiten rund um die Belagerung der Burg Weineck zu beseitigen. Stattdessen soll die Lektüre der folgenden Seiten zu weiteren Fragen anregen und einen Forschungsprozess in Gang setzen.

\section{Belagerung der Burg Weineck im Kampf zwischen geistlicher und welt- licher Macht}

Die Burg Weineck befand sich in Bozen am Virgl auf einer zweihundert Meter über dem Bozner Becken gelegenen Ausbuchtung des Kohlernbergs und stand an einer geostrategisch bedeutenden Schnittstelle dieses Gebietes. Am Fuße des Virgl fließt die Talfer in den Eisack. Wer im Mittelalter von Süden aus Bozen erreichen wollte, musste aufgrund der unpassierbaren Etsch an Virgl vorbei, um anschließend bei Kampill den Eisack zu überqueren. Selbiges galt für die Passage des oberen Etsch- und Eisacktals. Somit konn-

2 Josef Nössing, Weineck, in: Oswald Trapp (Hrsg.), Tiroler Burgenbuch, Bd. 8: Raum Bozen, Bozen 1989, S. 71-75, hier S. 73-74. 
ten die Weinecker, bedingt durch ihre Lage, den Nord-Süd Transit gut überwachen. ${ }^{3}$ Der Name der Burg leitet sich vom althochdeutschen Wort "Winni" ab und bedeutet "Gefolgschaftsfreund". Bewohnt wurde die Burg hauptsächlich von Ministerialen des Stifts Trient. ${ }^{4}$ Die Belagerung der Burg stand im engen Zusammenhang mit dem Konflikt zwischen Meinhard II. und den Bischöfen von Trient. Meinhard II. - auch „Schöpfer Tirols" genannt - erreichte die Territorialisierung und Machtausbreitung Tirols Richtung Süden durch jahrzehntelange Auseinandersetzungen mit dem Bistum Trient. Im Streit mit der Kirche erwies sich Meinhard II. als kluger Stratege und kaltblütiger Eroberer. Um die Macht der Kirche zurückzudrängen, war ihm vom Rechtsbruch bis zur Gewaltanwendung „jedes Mittel recht" die Übergabe von Lehen von Bischof Egno, vertrieb den Kirchenmann wenige Jahre darauf aus Trient und herrschte schlussendlich selbst über die Stadt. In den Jahren der Herrschaft über Trient ging er mit äußerster Härte gegen politische Gegner vor und griff zu Maßnahmen wie der Konfiszierung von Besitztümern oder der Anordnung von Gefängnisstrafen. ${ }^{6}$

Einige Male mussten der Papst und der römisch-deutsche König in die Auseinandersetzung zwischen Meinhard und der Kirche eingreifen. Diverse Interventionen König Rudolfs von Habsburg erscheinen aus heutiger Sicht jedoch als reine Formalakte. Ungeachtet dessen trieb Meinhard II. seine Expansionsambitionen im Herrschaftsgebiet von Trient vehement voran. Er hatte die Gunst des Königs durch seine militärische Unterstützung Rudolfs im Kampf gegen König Ottokar von Böhmen gewonnen, daneben wusste er aufgrund verwandtschaftlicher Bindungen und Gelddarlehen das Reichsoberhaupt auf seiner Seite.? 1277 überfiel Meinhard II. Bozen und Trient. Massive Zerstörungen in Bozen schüchterten die ansässige Bevölkerung dermaßen ein, dass sie sich Meinhard II. unterwarf. Um seine Stiftsstadt vor dem heranrückenden Landesfürsten zu verteidigen, suchte der Trienter Bischof Heinrich II. Hilfe im Süden und verbündete sich mit der Republik Padua und deren Städtebund. Bei Trient kam es zur Schlacht, aus der die Truppen Meinhards II. als Sieger hervorgingen. Die Vormacht Meinhards II. über das Trienter Gebiet war nun nicht mehr zu brechen. Auch unter dem Nachfolger Heinrichs II., Bischof Phillipp von Bonacolsi, setzten sich die Konfrontationen fort. Im Jahre 1295 erklärte Meinhard II. schließlich die Restitution aller jahrzehntelang erkämpften Kirchengüter. ${ }^{8}$

Nach mehreren Interdikten über das Land, wiederholtem Kirchenbann Meinhards II., zahlreichen Streitzügen und Verhandlungen war Trient zwar de jure kein Teil des Landesfürstentums, de facto blieb die Stiftsstadt aber in enger Abhängigkeit zur Grafschaft 106.

$4 \quad$ Nössing, Weineck, S. 71-72.

5 Michael Forcher, Kleine Geschichte Tirols, Innsbruck-Wien 2006, S. 26.

6 Franz Hammerl, Tirol. Des Reiches Südmark im Mittelalter, Leipzig 1939, S. 91-93.

7 Josef Riedmann, Das entscheidende Jahrhundert in der Geschichte Tirols (1259-1363), in: Südtiroler Landesmuseum Schloß Tirol/Tiroler Landesmuseum Ferdinandeum (Hrsg.), Eines Fürsten Traum. Meinhard II. - Das Werden Tirols, Katalog zur Tiroler Landesausstellung von Schloss Tirol und Stift Stams von 31.5.199531.10.1995, Dorf Tirol-Innsbruck 1995, S. 27-58, hier S. 41. 
Tirol. Mittels Verträgen über Schutz-, Wehr- und Steuerhoheiten sicherte sich der Tiroler Landesfürst die Macht über Trient und dessen Stiftsländereien. ${ }^{9}$ Die Burg Weineck blieb von der tobenden Auseinandersetzung lange Zeit unbehelligt. Vermutungen, Weineck musste als Sitz der Trienter Ministerialen ein strategisch wichtiges Ziel für Meinhard II. dargestellt haben, können somit nicht bestätigt werden. Während andere bischofstreue Einrichtungen in der Umgebung beseitigt wurden und die Weinecker für ihren Lehensherren Partei ergriffen, wurde Weineck bei der Zerstörung Bozens 1277 nicht in Mitleidenschaft gezogen. Warum Meinhard II. sich nicht gegen Weineck richtete, bleibt rätselhaft. Erst im Jahre 1292 beauftragte der Landesfürst Otto von Königsberg mit der Belagerung der Burg, die insgesamt 13 Wochen, vom 18. Juli bis zum 16. Oktober, dauerte und mit dem Sieg Meinhards II. endete. Das Adelsgeschlecht von Weineck bestand weiterhin fort, doch die Burg wurde zur Gänze abgetragen. ${ }^{10}$ Heute zeugen spärliche Überreste von der einstmals mächtigen Burganlage, daneben wird die Burg in nur einigen wenigen Überlieferungen erwähnt.." Eine dieser Quellen sind die Rechnungslegungen der Kanzlei Meinhards II., welche die Verpflegung der Belagerungsmannschaften dokumentieren.

\section{Die Küche hinter der Belagerung}

Vor der Analyse der Rechnung Jakob Hossers werden einige relevante Randinformationen dargeboten und in den historischen Kontext eingebettet. Meinhard II. war es gelungen, aus den heterogen beherrschten Gebieten an Etsch, Eisack und Inn ein einheitliches Territorium zu formen. Nach dem Historiker Christoph Haidacher basierte seine Strategie auf zwei Faktoren: Einerseits der brutalen Anwendung von Gewalt, andererseits der gezielten Nutzung von Geld als politisches Mittel. Dazu gehörten etwa die regelmäßige Investition in Liegenschaften, der Goldhandel oder Bankgeschäfte. Geld- bzw. finanzpolitische Vorhaben stärkten die machtpolitische Stellung Meinhards II. und füllten gleichzeitig die landesfürstlichen Kassen. Ohne einen funktionierenden Verwaltungsapparat hätte sich eine derartige Politik kaum bewerkstelligen lassen. ${ }^{12}$ Diese ausgeklügelte Verwaltungsorganisation ermöglichte es Meinhard II., das Land nachhaltig gegen verschiedene geistliche und weltliche Ansprüche zu verteidigen und seinen Machtbereich stetig zu erweitern. Ein Produkt ebendieser Organisation sind die älteren Tiroler Rechnungsbücher, die unter anderen die Rechnungen zur Belagerung von Weineck inkludieren.

9 Forcher, Kleine Geschichte, S. 26.

10 Nössing, Weineck, S. 73.

11 Zu den urkundlichen Erwähnungen: Hannes Obermair, Bozner Urkundenwesen des Mittelalters und die Gründung der städtischen Siedlung Bozen, in: Reimo Lunz (Hrsg.), Bozen von den Anfängen bis zur Schleifung der Stadtmauer (Berichte der internationalen Studientagung in Schloß Maretsch), Bozen 1991, S. 159-190, hier S. 176-177. Näheres zu dem Weinecker Geschlecht, Genealogie sowie dem Aufbau der Burg: Innerebner, Schloß Weinegg, S. 107-109.

12 Christoph Haidacher, Die älteren Tiroler Rechnungsbücher (IC. 277, MC. 8). Analyse und Edition, Bd. 1 (Tiroler Geschichtsquellen 40), Innsbruck 1993, S. 11-13. 


\section{Die älteren Tiroler Rechnungsbücher}

Diese Rechnungsbücher bzw. "Raitbücher" (von „raiten“, Mittelhochdeutsch für "zahlen",,'rechnen") stellen eine einzigartige Quelle dar. ${ }^{13}$ Wo anderorts in Europa von derartigen Dokumenten aus dem Spätmittelalter bloß Fragmente erhalten sind, überlieferte der Verwaltungsapparat des spätmittelalterlichen Tirols ein breites Repertoire an Quellenmaterial. Im Zeitraum zwischen 1288 und 1350 produzierte die Kanzlei der Tiroler Landesfürsten um die zwanzig Codices. ${ }^{14}$ Sie dokumentierten genauestens die Einnahmen und Ausgaben der öffentlichen Verwaltung, heutige Maßstäbe der Buchführung erfüllten sie allerdings nicht. Elisabeth Bamberger zufolge entsprechen die Tiroler Rechnungsbücher einer "laufende[n] Reihe von Rechnungsberichten lokaler Amtleute $[\mathrm{sic}]^{\prime \prime 15}$. Vereinzelte Überlieferungen von ähnlichen Dokumenten aus anderen römischdeutschen Ländern sind im Vergleich inhaltlich magerer und besitzen weniger Aussagekraft. ${ }^{16}$ Die Tiroler Rechnungsbücher halten den Gegenstand und den Grund einzelner Ausgabeposten fest und liefern somit Informationen über die (Verwaltungs-) Geschichte des Tiroler Raums und darüber hinaus. ${ }^{17}$ Im meinhardinischen Tirol war die Führung derartig genauer Aufzeichnungen notwendig, um die Einnahmen und Ausgaben im Überblick zu behalten. ${ }^{18}$ Aufgrund des Fehlens von landesübergreifenden Finanzbilanzen konnten die Rechnungsbücher eine Übersicht über die finanziellen Tätigkeiten der Gerichte, Zölle und Salinen generieren. ${ }^{19}$ Sie wiesen die von den Beamten gemachten Angaben nach, offensichtlich auch, um Veruntreuung zu verhindern. ${ }^{20}$

\section{Quittung einer Belagerung}

Zur Belagerung der Burg Weineck wurden sechs Rechnungen verfasst, wovon aber nur drei vollständig überliefert sind. Die Rechnungslegung des Küchenmeisters Jakob Hosser über die bei der Belagerung Weinecks aufgewendeten Lebensmittel berichtet über die gesamten 13 Wochen der Belagerung, während die anderen erhaltenen nur vier oder fünf Wochen abdecken. ${ }^{21}$ Seine Rechnung beginnt mit einer Kontexterzählung inklusive der Angabe des Datums und des Küchenmeisters: Am Freitagabend des 18. Juli 1292 soll Otto von Königsberg gemeinsam mit dem Burggrafen ${ }^{22}$ und dessen

13 Josef Riedmann, Die Rechnungsbücher der Tiroler Landesfürsten, in: Gabriel Silagi (Hrsg.), Landesherrliche Kanzleien im Spätmittelalter (Münchner Beiträge zur Mediävistik und Renaissance-Forschung 35), München 1984, S. 314-324, http://elec.enc.sorbonne.fr/cid/cid1983/art_13, eingesehen 5.8.2020.

14 Riedmann, Rechnungsbücher, S. 314-324.

15 Elisabeth Bamberger, Die Finanzverwaltung in den deutschen Territorien des Mittelalters (1200-1500), in: Zeitschrift für die gesamte Staatswissenschaft/Journal of Institutional and Theoretical Economics 77 (1922-23), Heft 1-2, S. 168-255, hier S. 218.

16 Otto Stolz, Der geschichtliche Inhalt der Rechnungsbücher der Tiroler Landesfürsten von 1288-1350 (Schlern Schriften 175), Innsbruck 1957, S. 5.

17 Ebd.

18 Haidacher, Rechnungsbücher, Bd. 1, S. 18.

19 Ebd

20 Bamberger, Finanzverwaltung, S. 218.

21 Christoph Haidacher, Die älteren Tiroler Rechnungsbücher (IC. 278, IC 279 und Belagerung von Weineck). Analyse und Edition (Tiroler Geschichtsquellen 40), Bd. 2, Innsbruck 1998, S. 15-16.

22 Burggraf (purgravio): Militärkommandant der Burg, Amt der Tiroler Grafschaft. 
Gesellschaft vor das Lager von Weineck getreten sein. ${ }^{23}$ Otto von Königsberg und der Burggraf sollen Hosser zum Küchenmeister berufen haben, weil er ihnen "gut passte ${ }^{\prime 24}$. Allen Anschein nach fanden sie Gefallen an seinen Kochkünsten. Anschließend folgt die genaue Rechnungslegung der aufgewendeten Lebensmittel. In der Regel wurden die Lebensmittel mitsamt den Mengenangaben und dem dafür bezahlten Geldbetrag notiert. Leider fehlt in der Auflistung häufig der Geldbetrag, was die Analyse erschwert. Hosser bezog die Nahrungsmittel zumeist von Konrad, dem Beschließer ${ }^{25}$ von Gries, oft aber auch vom Verwalter Gerold. Zweimal ist vom Verwalter Enna die Rede. ${ }^{26}$

Die Angabe der bezahlten Geldmengen erfolgte in der Rechnung mittels den Abkürzungen Ib. Ver, G, S und Ver. Im Nachtrag ist einmalig die Abkürzung $m$ zu finden. Die Tirolischen Münzen waren benannt nach ihrer Ursprungsstätte Verona, zu Deutsch Bern bzw. Welsch-Bern. Die Abkürzung $m$ steht für marca Veronesium bzw. Mark Berner, während /b. Ver. für libra Veronensium, dem Pfund Berner, steht. G meint Grossus, den Groschen oder Zwanziger bzw. Kreuzer. S steht in der Rechnung für Solidus, im Tirolischen Schilling. Dazu kommt die „kleinste" der tirolischen Münzen, der Veronenses bzw. Berner. Das Verhältnis der verschiedenen Münzen zueinander lautete wie folgt: Ein Mark entsprach zehn Pfund, zehn Pfund waren 120 Groschen, 120 Groschen waren zweihundert Schillinge und zweihundert Schillinge waren 2.400 Berner. Mark und Solidus galten nur als Rechnungsmünzen, auch das Pfund war zu diesem Zeitpunkt noch keine etablierte Münze. In Bezug auf die untersuchte Rechnung bedeutet dies, dass allein der Groschen und der Berner real im Umlauf waren. ${ }^{27}$

\section{Analyse der Rechnungslegung des Küchenmeisters Jakob Hosser}

Wie bereits im vorherigen Kapitel erwähnt, ist die Analyse der Rechnung nicht unproblematisch. Bei einigen Angaben fehlt die Mengenbezeichnung und/oder der verrechnete Preis der Ware. In der unten angeführten Tabelle 1 wurde trotz dieser Ungereimtheiten versucht, sämtliche eingekaufte Waren in einer Gesamtdarstellung zu vereinen und fünf Kategorien zuzuordnen. Um einen Überblick darüber zu geben, welche und vor allem wie viele Ressourcen eine Belagerung benötigte, wurden in der Tabelle (wo vorhanden) die Mengenangaben angeführt. In den eckigen Klammern ist das Mengenmaß angegeben. Insgesamt kommen in der Rechnung drei Maßeinheiten vor: das Maß, das Sterium und das Pazeiden. Alle drei sind Hohlmaße. Das Maß wird heute in der Regel nur noch für die Angabe einer Biermenge (1 Liter) verwendet. In der Vergangenheit variierte die Menge eines Maßes zwischen 0,8 und 2 Liter und wurde räumlich sowie zeitlich unterschiedlich verwendet. ${ }^{28}$ Das Sterium wird heute als Raummeter oder Ster hauptsächlich

\footnotetext{
23 Haidacher Rechnungsbücher, Bd. 2, S. 43-45.

24 Ebd.; Hosserius [...] bene constat: Siehe dazu die Übersetzung der lateinischen Rechnung ins Deutsche (Anlage).

25 Ebd.; Beschließer (claviero): Amt der Tiroler Grafschaft.

26 Ebd.

27 Ferdinand Kogler, Übersicht über das Münzwesen Tirols bis zum Ausgang des Mittelalters, in: Finanz Archiv/Public Finance Analysis 19 (1902), Heft 2, S. 133-135, hier S. 133.

28 Mass, in: Historisches Lexikon der Schweiz, aktualisiert 29.10.2009, https://hls-dhs-dss.ch/de/articles/ 014198/2009-10-29/, eingesehen 7.4.2021.
} 
in der Holzwirtschaft verwendet und entspricht einem Kubikmeter. Ein Ster Mehl würde beispielsweise $715 \mathrm{~kg}$ Mehl entsprechen. ${ }^{29}$ Einmal wird in der Rechnung die Einheit Pazeiden verwendet, welche hauptsächlich für Wein, in der Rechnung allerdings für Essig, verwendet wurde. Ein Pazeiden ergibt etwa 6,43 bis 6,85 Liter. ${ }^{30}$ Fehlt die Maßeinheit, handelt es sich in der Tabelle um die Stückzahl. Wenn in der Rechnung keine Mengenangaben gemacht wurden, sind die Waren in ihrem Geldwert wiedergegeben. Deren Größenordnung (Ib Ver, G, S) ist hierbei in eckigen Klammern angeführt.

\begin{tabular}{|c|c|c|c|c|c|c|c|c|c|c|c|c|c|c|}
\hline & Woche & 1 & 2 & 3 & 4 & 5 & 6 & 7 & 8 & 9 & 10 & 11 & 12 & 13 \\
\hline \multirow{9}{*}{$\begin{array}{l}\text { Fleisch } \\
\text { und Fisch }\end{array}$} & Fische [G] & & & 28 & & 20 & 17 & & & & & & & \\
\hline & Fische [S] & & & & 35 & & & & & & & & & \\
\hline & frische Rinder & 8 & 10 & 5 & 6 & 3 & 4,25 & 5 & 4 & & 10 & 11 & 10 & 12 \\
\hline & Schafe & & & 33 & 10 & 20 & 12 & 26 & & & & & & \\
\hline & Schafe [S] & & & 5 & & & & & & & & & & \\
\hline & getrocknete Schafe & & & & & & & & & 3 & & & & \\
\hline & Schultern & & & & 4 & & & & & & & & & \\
\hline & Schweine & & & & & & 0,5 & & & & & & & \\
\hline & getrocknete Schweineschinken & 6 & 5 & 5 & 4 & 5 & 4 & 3 & & & & & & \\
\hline \multirow{5}{*}{$\begin{array}{c}\text { Tier- } \\
\text { produkte }\end{array}$} & Schmalz [Maß] & & & & & & & & & & & & 1 & \\
\hline & \begin{tabular}{|l|} 
Schmalz [G] \\
\end{tabular} & & & 4 & & & 4 & & & & & & & \\
\hline & Schmalz [S] & & & & 13 & 12 & 4 & & & & & & & \\
\hline & Eier & 150 & 100 & & 170 & 100 & 100 & & & & & & & \\
\hline & Käse & 600 & 350 & 160 & 245 & 350 & 250 & 200 & 200 & 300 & 300 & 110 & 300 & 250 \\
\hline \multirow{5}{*}{$\begin{array}{l}\text { Getreide } \\
\text { und } \\
\text { Hülsenfrüchte }\end{array}$} & Bohnen [Maß] & 7 & 4 & 6,5 & 10 & & & & & & & & & \\
\hline & Griesmehl [Sterium] & & & & & 1 & & & & & & & & \\
\hline & grobes Mehl [Sterium] & & & & & 4 & & & & & & & & \\
\hline & Mehl [Sterium] & & & & 4,5 & 4 & & & & & & & & \\
\hline & Roggen [Sterium] & & & & & 1 & & & & & & & & \\
\hline \multirow{4}{*}{$\begin{array}{c}\text { Würz- } \\
\text { und } \\
\text { Konservierungs- } \\
\text { mittel }\end{array}$} & Essig [Pazeiden] & & & & & & & & & 3,5 & 3,5 & & & \\
\hline & Kräuter [G] & & & & & & 3 & & & & & & & \\
\hline & Kräuter [S] & & & & & & 13 & & & & & & & \\
\hline & Pfeffer und Safran [S] & 24 & 24 & 24 & 24 & 24 & 24 & & & & & & & \\
\hline \multirow{5}{*}{ Sonstiges } & Diverses [1b. Ver.] & & & & & & 12 & 6,5 & 33,5 & 44 & 10 & 28 & 29 & 18 \\
\hline & Diverses [G] & & & & & & & & 4 & 8 & & 9 & & -2 \\
\hline & Diverses [S] & & & & & & & 4 & & & 15 & & & \\
\hline & Diverses [Ver] & & & & & & & & 8 & 6 & 4 & 10 & & \\
\hline & Tischgeschirr [S] & 30 & & & & & & & & & & & & \\
\hline
\end{tabular}

Abbildung 1: Tabellarische Darstellung der Rechnung (vom Autoren erstellt; Quelle: Haidacher ${ }^{31}$ )

Auf den ersten Blick sticht hervor, dass sich die meisten Werte auf die ersten Wochen und damit den Beginn der Belagerung konzentrieren. Ab der sechsten Woche wurden statt der Mengen- sowie Geldangaben der einzelnen Güter lediglich Naturalien aufgezählt und der dafür bezahlte Geldbetrag hinterlegt. Jene Einträge, die die Geldsumme für verschiedene Waren angeben, werde in der Tabelle in der Kategorie "Diverses" subsumiert. Eine Untersuchung der Mengen ist somit nur bis zur sechsten bzw. siebten Woche sinnvoll. In den ersten fünf Wochen wurde der Großteil der Waren über den Beschließer Konrad abgewickelt, mit fortschreitender Belagerung nahm Hosser aber zunehmend Lebensmittel vom Verwalter Gerold ab. Wenn der Küchenmeister Lebens-

29 Ster, in: Holzlexikon Holz Kars GmbH, o. D., https://holz-kahrs.de/lexikon/ster, eingesehen 7.4.2021.

30 Pazeiden, in: Austria Forum, aktualisiert 12.10.2019, https://austria-forum.org/af/AustriaWiki/Pazeiden, eingesehen 24.9.2020.

31 Haidacher, Rechnungsbücher, Bd. 2, S. 43-44. 
mittel von Gerold bezog, weist die Abrechnung Lücken auf. Im Gegensatz dazu wurde die Rechnungslegung bei Konrad sehr präzise geführt. Auch bei den Geschäften mit dem Verwalter Enna wurden Mengenangaben genannt. Relativ vage und intransparent erscheint die Buchführung in der elften, zwölften und 13. Woche. Hier finden sich in den Summenaufzählungen unpräzise Vermerke wie „und anderes zum Kochen“ bzw. „und anderes für die Küche ${ }^{\prime 32}$. Naheliegende Vermutungen, es habe sich dabei um Kräuter oder Gewürze gehandelt, sind unzutreffend, da jene Produkte separat in der Aufstellung geführt wurden. Warum die Abrechnung gegen Ende der Belagerung und insbesondere beim Bezug der Waren vom Verwalter Gerold dermaßen ungenau und oberflächlich dargelegt wurde, ist aus heutiger Sicht kaum nachvollziehbar. Hosser und Gerold der Veruntreuung von Geldern zu beschuldigen, wäre aufgrund der unsicheren Beweislage aber keine plausible Erklärungsvariante.

Die Gesamtdarstellung (Tabelle 1) verdeutlicht, dass manche Waren regelmäßig bezogen wurden, während es sich bei anderen um Einzelkäufe handelte. Deutlich sticht hierbei das Tischgeschirr hervor, welches in der ersten Woche "auf Geheiß des Herrn Otto" gekauft wurde. ${ }^{33}$ Weitere Beispiele wären vier Schultern (von welchem Tier ist nicht notiert) in der vierten, ein halbes Schwein in der sechsten oder drei getrocknete Schafe in der neunten Woche. ${ }^{34}$ Bei den Schultern und den getrockneten Schafen könnte es sich um kulinarische Besonderheiten gehandelt haben, die ansonsten schwer oder nur teuer zu bekommen waren. Demgegenüber stehen Rinder, Käse, Eier, Schmalz, Pfeffer und Safran, welche die gesamte Belagerung hindurch nahezu jede Woche gelistet wurden. ${ }^{35}$ Manche Lebensmittel wurden hingegen gelegentlich bzw. nur zu gewissen Perioden gekauft. So wurden Schafe ab der dritten Woche bis zum Ende der Belagerung (mit Ausnahme der elften Woche) angeschafft, Fische standen in acht der 13 Belagerungswochen auf dem Speiseplan. Interessant erscheint der Wechsel von Bohnen zu Getreide: In den ersten vier Wochen, vom 21. Juli bis zum 24. August, wurden wöchentlich zwischen sechseinhalb und zehn Maß Bohnen erworben, von der fünften Woche bis zum Ende der Belagerung jedoch kein einziges Maß mehr. Gleichzeitig wurden in den ersten drei Wochen kein Getreide bzw. Mehl bestellt. Mit relativ großer Wahrscheinlichkeit ist dies auf die Erntezeit von Bohnen zurückzuführen, da die Ackerbohne bis Ende Juni/Anfang Juli geerntet wird. ${ }^{36}$ Rund um diesen Zeitraum war die Hülsenfrucht wahrscheinlich um einiges billiger zu bekommen als Getreide, weshalb sie sich als günstige, sättigende und nahrhafte Alternative anbot. In der vierten Woche erwarb Hosser große Mengen Getreide und Mehl, die in der neunten Woche langsam versiegten. Vermutlich wurde Mehl ab diesem Zeitpunkt nur mehr in kleineren Mengen angeschafft, da es bis zum Ende der Belagerung wöchentlich

\footnotetext{
32 Haidacher, Rechnungsbücher, Bd. 2, S. 43-45; siehe Anlage.

33 Ebd., S. 43-44.

34 Auch in den Summeneinträgen (in der Tabelle unter "Diverses") wurden diese Lebensmittel nicht genannt.

35 Eier, Schmalz, Pfeffer und Safran waren ab der siebten Woche in den Summeneinträgen vermerkt.

36 Plantura Magazin (Hrsg.), Ackerbohne. Alles zum Anbau im eigenen Garten, o. D., https://www.plantura. garden/gartentipps/gemuseratgeber/ackerbohne-alles-zum-anbau-im-eigenen-garten\#: :text=den\%20 schleimigen\%20Schnecken.-,Ackerbohne\%20ernten\%20und\%20lagern,die\%20Aussaat\%20spät\%20erfolgt\%20 ist., eingesehen 4.8.2020.
} 
angeführt wurde. ${ }^{37}$ Ähnliches galt für Kräuter, die von der sechsten Woche, also ab dem 25. August, bis zum Ende der Belagerung (mit Ausnahme der zwölften Woche) erfasst sind. Um welche Kräuter es sich dabei konkret handelte, gibt die Rechnung nicht preis. Unwahrscheinlich ist, dass die Kräuter für den regulären Küchengebrauch gedacht waren, denn in den ersten fünf Wochen sind keinerlei Hinweise auf Kräuter in den Aufzeichnungen zu finden. Womöglich fanden sie Verwendung in heilenden Tees oder Suppen, da der Herbst und damit die kalte Jahreszeit bevorstand. In diesem Kontext ist auch Essig zu nennen: In der neunten und zehnten Woche der Belagerung, also in der dritten und vierten Septemberwoche, wurden etwa 45 Liter Essig gekauft. Im Mittelalter war das Wissen um die nützliche Wirkung von Essig oder ähnlichen Säuren im Zusammenhang mit der Lebensmittelkonservierung weit verbreitet. ${ }^{38}$ Bereits um 5000 v. Chr. wurden Säuren zu diesem Zweck verwendet. ${ }^{39}$ Höchstwahrscheinlich besorgte Hosser den Essig, um wertvolle Lebensmittel für den Winter haltbar zu machen. Diese Vermutung untermauert die ab derselben Woche beginnende regelmäßige Anschaffung von Salz, das ebenfalls als Konservierungsmittel diente.

Die Betrachtung der einzelnen Lebensmittel lässt jedenfalls folgende Rückschlüsse zu: In den 13 Wochen werden insgesamt zwanzig verschiedene Waren in der Rechnungslegung genannt. Die folgende Darstellung ordnet die Waren aus Tabelle 1 fünf Kategorien zu, wobei die Kategorie „Sonstiges" keine Lebensmittel beinhaltet. Das untenstehende Tortendiagramm vergleicht die einzelnen Kategorien miteinander (die Zahlen neben den Kategorien entsprechen Prozentangaben):

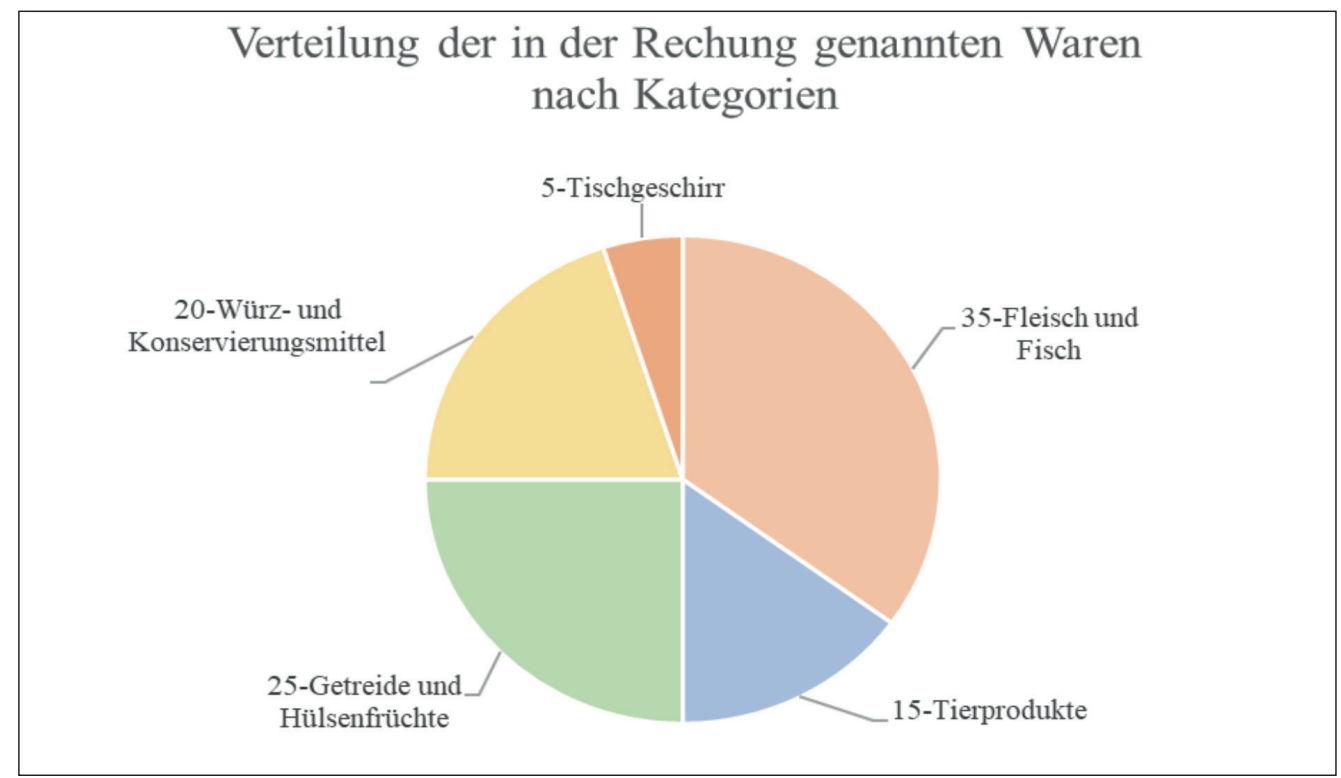

Abbildung 2: Verteilung nach Kategorien (vom Autor erstellt; Quelle: Haidacher ${ }^{40}$ )

37 Genaue Mengen sind aus dem Summeneintrag nicht ersichtlich.

38 Erich Lück, Chemische Lebensmittelkonservierung. Stoffe - Wirkungen - Methoden, Berlin-Heidelberg u. a. 1986, S. 133.

39 Ebd.

40 Haidacher, Rechnungsbücher, Bd. 2, S. 43-44 
Mit 35 Prozent aller Waren nimmt die Kategorie „Fleisch und Fisch" einen beachtlichen Teil der beschafften Lebensmittel ein. Um welche Art von Fisch es sich dabei handelte, wurde in der Rechnung nicht näher spezifiziert. Die vorliegenden Daten legen nahe, dass in Hossers Küche eine große Diversität an Fleischprodukten herrschte. Wird zur Kategorie "Fleisch und Fisch" die Kategorie "Tierprodukte" (15 Prozent) hinzugezählt, ergibt sich, dass die Hälfte aller in der Rechnung erwähnten Nahrungsmittel tierischen Ursprungs war. Dieser hohe Wert ist beachtlich, aber nicht überraschend. Fleisch war im mittelalterlichen Mitteleuropa für die Nahrungsmittelversorgung von großer Bedeutung. Je nach Schätzung sollen die Menschen des Spätmittelalters jährlich etwa 50 bis 100 kg Fleisch pro Kopf konsumiert haben. ${ }^{41}$ Für die einfache Bevölkerung dürften 50 kg am ehesten zutreffen bzw. bereits die Obergrenze darstellen. Im Falle der Belagerer von Weineck sind 100 kg als wahrscheinlicher anzunehmen, da Söldnern in ihrer Dienstzeit eine nahrhafte Verpflegung zustand und sie einen Teil ihres Soldes in Naturalien erhielten. ${ }^{42}$ Zum Vergleich: In Österreich wurden im Jahr 2018 pro Person durchschnittlich 64 kg Fleisch verzehrt. ${ }^{43}$ Die zweitgrößte Kategorie "Getreide und Hülsenfrüchte" macht 25 Prozent der in der Rechnungslegung angeführten Waren aus. Mit zwanzig Prozent besitzen die „Würz- und Konservierungsmittel" ebenfalls einen beachtlichen Anteil an den angeschafften Waren.

Abschließend versucht das folgende Säulendiagramm, einen genaueren Eindruck über die Größenverhältnisse zu liefern. Es zeigt fünf Lebensmittel, die in den ersten sieben Wochen regelmäßig eingekauft wurden. Bei allen Angaben handelt es sich um die Stückzahl.

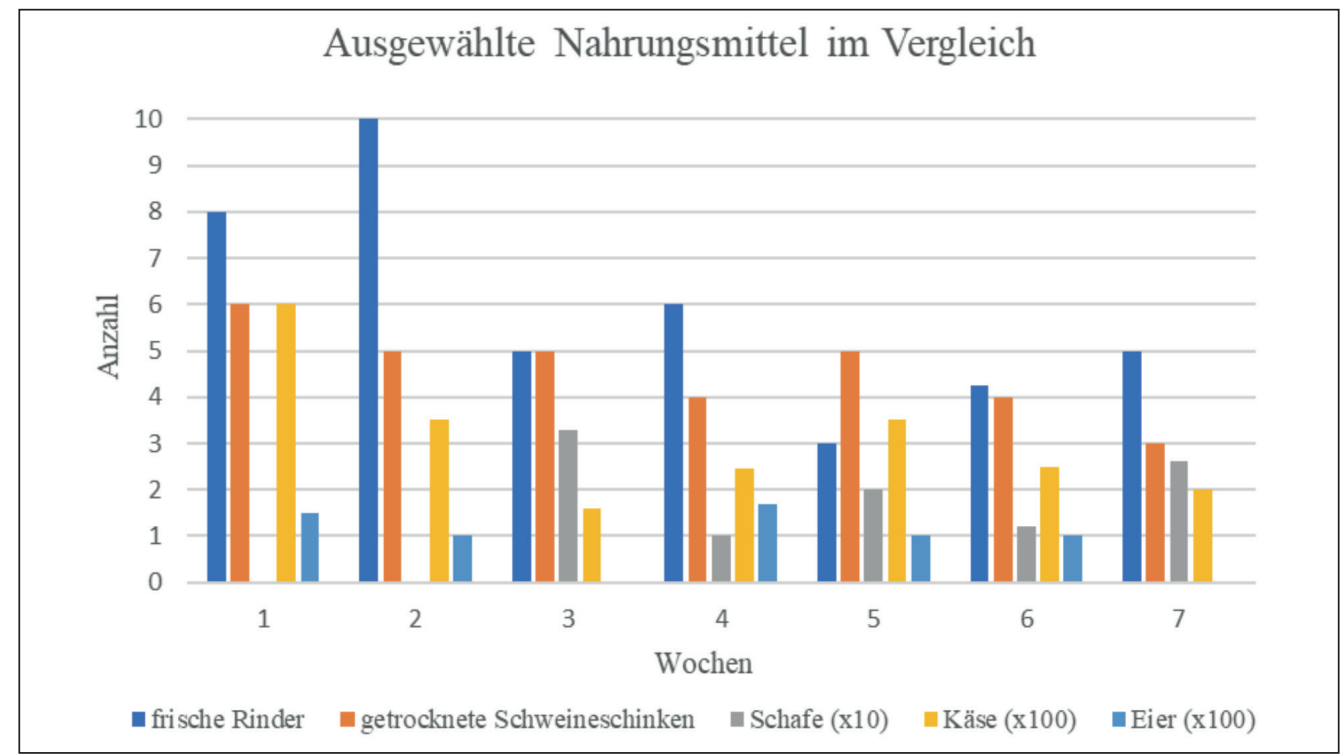

Abbildung 3: Mengenvergleich (vom Autoren erstellt; Quelle: Haidacher ${ }^{44}$ )

41 Ernst Schubert, Essen und Trinken im Mittelalter, Darmstadt 2006, S. 106; Hans Jürgen Teuteberg/Günter Wiegelmann, Nahrungsgewohnheiten in der Industrialisierung des 19. Jahrhunderts, Münster 1995, S. 99.

42 Schubert, Essen und Trinken, S. 106.

43 oesterreich.ORF.at (Hrsg.), Fleischkonsum im Vorjahr leicht gestiegen, 29.8.2019, https://oesterreich.orf.at/ stories/3010570/, eingesehen 4.8.2020.

44 Haidacher, Rechnungsbücher, Bd. 2, S. 43-44. 
Der Abbildung lässt sich entnehmen, dass in den ersten sieben Wochen 41 ganze Rinder und ein Viertel eines Tieres gekauft wurden. Die Anzahl der Rinder variierte von Woche zu Woche zwischen drei und zehn Stück. Im Durchschnitt wurden in den sieben Wochen wöchentlich rund sechs Stück angeschafft. Ein ähnliches Bild ergibt der Blick auf die übrigen Lebensmittel: Sie sind allesamt Schwankungen ausgesetzt. Mit 32 Stück ist die Summe der angeschafften Schweineschinken etwas niedriger als die der Rinder. Der Durchschnitt beträgt viereinhalb Stück Schweineschinken pro Woche. Allerdings muss in diesem Diagramm die Menge der Schafe mit dem Faktor zehn, die der Eier und der Käse mit dem Faktor hundert multipliziert werden, was der Darstellungen derartig variierender Mengen in einem gemeinsamen Diagramm geschuldet ist. Somit wurden in der ersten Woche sechshundert Stück Käse erworben, durchschnittlich wurden pro Woche 308 Stück beschafft. Die Summe der ersten sieben Wochen ergibt 2.155 Stück Käse. Im Gegensatz dazu liegt der Durchschnitt der wöchentlich angeschafften Eier bei 103 Stück, insgesamt wurden in diesen sieben Wochen 620 Eier besorgt. Der Ankauf von Schafen begann erst in der dritten Woche. Hosser schaffte 33 Stück davon an. Mit einem Mittelwert von zwanzig wurden in den fünf Wochen (Wochen 3-7) 101 ganze Schafe gekauft.

Absolut gesehen wurde in der ersten Woche von den hier dargestellten vier Waren eine beachtliche Menge erworben. In keiner der übrigen Wochen wurden so viele getrocknete Schweineschinken und Käse gekauft. Bei den Rindern belegt die erste Woche den zweithöchsten Wert. Vermutungen, die Lebensmittel seien zwar gekauft, aber nicht unmittelbar im Anschluss konsumiert worden, widerlegen die gewonnenen Daten klar: Von den acht Rindern in der ersten Woche scheinen in der zweiten Woche keine mehr vorrätig gewesen zu sein, sodass Hosser erneut zehn Stück einkaufte. Gegen einen Vorratseinkauf spricht auch die kurze Haltbarkeit. Die Konservierung von so großen Mengen wäre zu aufwendig gewesen. Davon abgesehen besorgte Hosser erst ab der neunten Woche regelmäßig Konservierungsmittel wie Salz oder Essig. Somit wurden die Lebensmittel - zumindest in den ersten Wochen - zeitnah konsumiert. Die Gesamtsumme der Verpflegung der Belagerung sowie des Abbruchs der Burg kostete die landesfürstliche Kasse zweihundert Mark, achtzig Pfund und zwölf Groschen. ${ }^{45} \mathrm{Im}$ Vergleich: Meinhard II. soll jährlich über tausend Mark für den Erwerb von Liegenschaften ausgegeben haben. ${ }^{46}$

\section{Fazit}

Die Burg Weineck am Virgl bei Bozen war zweifelsohne ein strategisch bedeutender Außenposten des Hochstifts Trient. Im Kampf um die Herrschaft über die Bozner und Trientner Gebiete war es angesichts von zahlreichen Überfällen, Drohungen und Schlachten seit 1258 - insbesondere aber nach der Zerstörung von Bozen im Jahre 1277 - nur eine Frage der Zeit, bis es zu einer Belagerung der Burg durch den Tiroler Landesfürsten Meinhard II. kam. Der 13-wöchigen Belagerung folgte die Zerstörung

45 Nössing, Weineck, S. 74.

46 Haidacher, Rechnungsbücher, Bd. 1, S. 12. 
der Burg. Aus dem Konflikt mit den Bischöfen von Trient ging Meinhard II. als Sieger hervor. Seinen Gebiets-, Vermögens- und Machtzuwachs konnte er durch eine ausgeklügelte Verwaltungsorganisation sichern und entwickelte hierfür ein für das damalige Europa einzigartiges Buchführungssystem. Mit den landesfürstlichen Rechnungsbüchern war die Verwaltung Meinhards II. in der Lage, die laufenden Einnahmen und Ausgaben sowie die eigenen Beamten zu kontrollieren. Aus dieser Verwaltungstätigkeit entsprang die Rechnungslegung des Küchenmeisters Jakob Hosser, eine der drei erhaltenen Rechnungen zur Belagerung Weinecks. Die Approvisation dokumentiert mehr oder weniger genau, von wem Hosser welche Waren in welcher Menge und zu welchem Preis erhielt. Obwohl die Rechnungslegung in Hinblick auf die Angabe von Mengen-, Maß- und Geldeinheiten nicht einheitlich ist, präsentierte die vorliegende Arbeit eine statistische Analyse der angekauften Waren. Waren, wie etwa getrocknete Schafe, wurden als einmalige, Käse oder Eier hingegen als regelmäßige Einkäufe identifiziert. Darüber hinaus wurden Fische als gelegentliche und Konservierungsmittel als periodische Käufe klassifiziert. Der Wechsel von Bohnen zu Weizen- und Roggenprodukten war vermutlich saisonal begründet, Mehl wurde ab einem gewissen Zeitpunkt in größeren Mengen beschafft. Das Kaufverhalten variierte sowohl je nach Jahreszeit als auch punktuell.

Zudem wurden insgesamt zwanzig verschiedene Waren kategorisiert: Die Kategorie "Fleisch und Fisch" war in der Küche Hossers am häufigsten vertreten, gefolgt von "Getreide und Hülsenfrüchten". Auf Platz drei lagen „Würz- und Konservierungsmittel”, während "Tierprodukte" den letzten Platz einnahmen. Die Hälfte der in den Rechnungen erwähnten Lebensmittel waren tierische Erzeugnisse bzw. Lebendtiere. Alles in allem gab es in Hossers Küche eine große Diversität an Nahrungsmitteln und dadurch konnten die Mahlzeiten der Belagerungstruppen abwechslungsreich gestaltet werden. Im Mengenvergleich wird deutlich, welche Dimension der Lebensmittelbedarf der Belagerer erreichte. Im Gegensatz zu heute verwertete die mittelalterliche Küche sämtliche Teile eines Tieres, wodurch die Zahlen noch eindrucksvoller werden. ${ }^{47}$ Die Darstellung führt vor Augen, dass die Einkäufe von Lebensmitteln von Woche zu Woche schwankten und wahrscheinlich von dem zur Verfügung stehenden Angebot und/oder Hossers Speiseplan abhingen. In Bezug auf die Leitthese der Arbeit ergeben die Erkenntnisse damit ein ambivalentes Bild: Die Approvisation wurde von Hosser gut verwaltet, der zweite Teil der Ausgangsthese wurde also verifiziert. Der erste Teil muss allerdings verworfen werden. Keinesfalls kann die Küche Hossers als nicht abwechslungsreich gelten, da er viele verschiedene Nahrungsmittel anschaffen ließ, mit denen er einen vielfältigen Speiseplan entwerfen konnte.

Wie einleitend angedeutet, bleiben noch zahlreiche Fragen und Forschungsansätze zur Belagerung Weinecks offen. Tiefergreifende Mengenanalysen der bezogenen Lebensmittel sind nötig, um die Ausmaße des Belagerungsaufwandes exakter fassen zu können. Ferner wäre es interessant und für eine umfassende Durchsicht notwendig, die anderen überlieferten Rechnungen miteinzubeziehen. Auch Geldwerte und Preise 
der Lebensmittel könnten bei zukünftigen größer angelegten Untersuchungen miteingebunden werden.

\section{Literatur und Quellen}

Bamberger, Elisabeth, Die Finanzverwaltung in den deutschen Territorien des Mittelalters (1200-1500), in: Zeitschrift für die gesamte Staatswissenschaft/Journal of Institutional and Theoretical Economics 77 (1922-23), Heft 1-2, S. 168-255.

Forcher, Michael, Kleine Geschichte Tirols, Innsbruck-Wien 2006.

Haidacher, Christoph, Die älteren Tiroler Rechnungsbücher (IC. 277, MC. 8). Analyse und Edition (Tiroler Geschichtsquellen 40), Bd. 1, Innsbruck 1993.

Ders., Die älteren Tiroler Rechnungsbücher (IC. 278, IC 279 und Belagerung von Weineck). Analyse und Edition (Tiroler Geschichtsquellen 40), Bd. 2, Innsbruck 1998.

Hammerl, Franz, Tirol. Des Reiches Südmark im Mittelalter, Leipzig 1939.

Innerebner, Georg, Schloß Weinegg und die Weinegger, in: Der Schlern 29 (1955), Heft 1, S. 104-117.

Kogler, Ferdinand, Übersicht über das Münzwesen Tirols bis zum Ausgang des Mittelalters, in: Finanz Archiv/Public Finance Analysis 19 (1902), Heft 2, S. 133-135.

Lück, Erich, Chemische Lebensmittelkonservierung. Stoffe - Wirkungen - Methoden, Berlin-Heidelberg u. a. 1986.

Mass, in: Historisches Lexikon der Schweiz, aktualisiert 29.10.2009, https://hls-dhs-dss. ch/de/articles/014198/2009-10-29/, eingesehen 7.4.2021.

Nössing, Josef, Weineck, in: Oswald Trapp (Hrsg.), Tiroler Burgenbuch. Bd. 8: Raum Bozen, Bozen 1989, S. 71-75

Obermair, Hannes, Bozner Urkundenwesen des Mittelalters und die Gründung der städtischen Siedlung Bozen, in: Reimo Lunz (Hrsg.), Bozen von den Anfängen bis zur Schleifung der Stadtmauer (Berichte der internationalen Studientagung in Schloß Maretsch), Bozen 1991, S. 159-190.

oesterreich.ORF.at (Hrsg.), Fleischkonsum im Vorjahr leicht gestiegen, 29.8.2019, https://oesterreich.orf.at/stories/3010570/, eingesehen 4.8.2020.

Pazeiden, in: Austria Forum, aktualisiert 12.10.2019, https://austria-forum.org/af/ AustriaWiki/Pazeiden, eingesehen 24.9.2020.

Plantura Magazin (Hrsg.), Ackerbohne. Alles zum Anbau im eigenen Garten, o. D., https://www.plantura.garden/gartentipps/gemuseratgeber/ackerbohne-alleszum-anbau-im-eigenen-garten\#: :text=den\%20schleimigen\%20Schnecken.,Ackerbohne\%20ernten\%20und\%20lagern,die\%20Aussaat\%20spät\%20erfolgt\%20ist., eingesehen 4.8.2020. 
Riedmann, Josef, Die Rechnungsbücher der Tiroler Landesfürsten, in: Gabriel Silagi (Hrsg.), Landesherrliche Kanzleien im Spätmittelalter (Münchner Beiträge zur Mediävistik und Renaissance-Forschung 35), München 1984, S. 314-324, http://elec.enc. sorbonne.fr/cid/cid1983/art_13, eingesehen 5.8.2020.

Ders., Das entscheidende Jahrhundert in der Geschichte Tirols (1259-1363), in: Südtiroler Landesmuseum Schloß Tirol/Tiroler Landesmuseum Ferdinandeum (Hrsg.), Eines Fürsten Traum. Meinhard II. - Das Werden Tirols, Katalog zur Tiroler Landesausstellung von Schloss Tirol und Stift Stams von 31.5.1995-31.10.1995, Dorf Tirol-Innsbruck 1995, S. 27-58.

Schubert, Ernst, Essen und Trinken im Mittelalter, Darmstadt 2006.

Ster, in: Holzlexikon Holz Kars GmbH, o. D., https://holz-kahrs.de/lexikon/ster, eingesehen 7.4.2021.

Stolz, Otto, Der geschichtliche Inhalt der Rechnungsbücher der Tiroler Landesfürsten von 1288-1350 (Schlern Schriften 175), Innsbruck 1957.

Teuteberg, Hans Jürgen/Günter Wiegelmann, Nahrungsgewohnheiten in der Industrialisierung des 19. Jahrhunderts, Münster 1995.

\section{Anlage: Übersetzung der Lateinischen Rechnung ins Deutsche ${ }^{48}$}

Rechnungslegung des Küchenmeisters Jakob Hosser über die bei der Belagerung der Burg Weineck aufgewendeten Lebensmittel - 1292 VII 18 - X (Mitte), Weineck (TLA, HS. 392. Fol. 2-4) 49

„Im Jahre des Herrn 1292 am Freitagabend nach dem Tag der Heiligen Margarete (18. Juli) kam der Herr Otto von Königsberg gemeinsam mit dem Burggrafen und dessen Gesellschaft vor das Lager Weinek. Dort wurde Hosser als Küchenmeister berufen, weil er dem Herrn Otto und dem Burggrafen gut passte. Er nahm am neunten Tag für die Familie zur Küche von Konrad, Beschließer von Gries, 6 getrocknete Schweineschinken, 8 frische Rinder, 7 Maß Bohnen ${ }^{50}, 600$ Käse auf. Auch vom Verwalter für Pfeffer und Safran $^{51} 24$ S, 150 Eier für 18 S. Auch für das Tischgeschirr auf Geheiß des Herrn Otto 30 S.

Auch in der zweiten Woche zahlte Hosser selbst für die Küche von Konrad, Beschließer von Gries, 5 getrocknete Schweineschinken, 10 Rinder, 4 Maß Bohnen, 350 Käse, 100 Eier für 12 S, vom Verwalter Gerold für Pfeffer und Safran 24 S.

Auch in der dritten Woche besorgte Hosser derselben Familie des Beschließer Konrad 5 Schweineschinken, 5 Rinder, 6 1⁄2 Maß Bohnen, 33 frische Schafe, 150 Käse. Auch in derselben [Anm.: Woche] vom Verwalter 10 Käse, Schmalz ${ }^{52} \mathrm{G} 4$, für Schafe S 5, für Fische G 28, für Pfeffer und Safran S 24.

\footnotetext{
48 Dem Kollegen Andreas Wieser möchte ich für die große Hilfe bei der Übersetzung herzlich danken.

49 Originale Rechnung: Haidacher, Rechnungsbücher, Bd. 2, S. 43-45.

50 "Fabe", vermutlich „fabae", also Bohnen

51 "Crocho", vermutlich „crocus" bzw. „croceus”, also Safran.

52 "Sagimen": Haidacher, Rechnungsbücher, Bd. 2, S. 587.
} 
Auch in der vierten Woche besorgte Hosser zur Küche zuerst von Konrad, dem Beschließer von Gries, 4 Schweineschinken, 6 Rinder, 10 Schafe, 10 Maß Bohnen, 225 Käse. Auch in derselben vom Verwalter in Gries 20 ganze Käse für ihn, 170 Eier für $S$ 20, Schmalz S 13, 1/2 Ster Mehl für S 7, in Fische S 35, für Pfeffer und Safran S 24. Auch in derselben vom Verwalter von Enna 4 Ster Mehl, 4 Schultern.

Auch in der fünften Woche besorgte Hosser für die Küche zuerst von Konrad, dem Beschließer von Gries, 2 lebende Rinder, 5 Schweineschinken, 1 Ster Roggen, 350 Käse. In derselben vom Verwalter 1 Rind für 5 Veroneser Denar, 20 lebende Schafe, für jedes beliebige S 16, für Fische S 20, Schmalz S 12, 1 Ster Grießmehl für S 14, 100 Eier für S 12, für Pfeffer und Safran S 24. In derselben vom Verwalter von Enna 4 Ster Mehl, 4 Ster grobes Mehl.

Auch in der sechsten Woche besorgte Hosser für die Küche des Beschließers Konrad 4 Schweineschinken, 250 Käse, $1 / 2$ lebendes Schwein von den Gütern der Herren von Weineck. Auch in derselben am Sonntag vom Verwalter 1 Rind, 2 Schafe für 8 1/2 Veroneser Denar, für Kräuter S 2. Auch am Montag 1 1/4 Rinder und 3 Schafe für 8 1 1/2 Veroneser Denar, für Kräuter S 3. Auch am Dienstag für Rinder- und Schafsfleisch Veroneser Denar 12. Auch am Mittwoch 1 Rind für Ib. Ver. 5 S 5, 4 Schafe für g 40, für Kräuter S 5. Auch am Donnerstag 1 Rind für S 55, 3 Schafe für g 30, für Kräuter S 3. Auch am Freitag für Fische S 10, Schmalz S 5, 50 Eier für S 5 1/2, für Kräuter g 1, für Pfeffer und Safran S 24. Auch am Samstag für Fisch g 7, 50 Eier für S 5 1⁄2, Schmalz g 4, für Kräuter $g 2$.

Auch in der siebten Woche erhielt Hosser für die Küche von dem Beschließer Konrad 3 Schweineschinken, 5 Rinder, 26 Schafe, 200 Käse. In derselben vom Verwalter Gerold in Fische, Schafe, Schmalz, Pfeffer und Safran, Kräuter Ib 6 1⁄2 S 4.

Auch in der achten Woche besorgte Hosser für die Küche des Beschließers Konrad 4 Rinder, 200 Käse. In derselben vom Verwalter für Rinder- und Schafsfleisch, Fische, Schmalz, Kräuter, Eier, Salz, Pfeffer und Safran und anderes Veroneser Denar 33 1⁄2 $g 4$ Ver. 8.

Auch in der neunten Woche erhielt und besorgte Hosser für die Küche vom Beschließer Konrad 3 getrocknete Schafe, 300 Käse, 3 1⁄2 Pazeiden Essig. In derselben vom Verwalter für Rinder- und Schafsfleisch, Fische, Schmalz, Kräuter, Eier, Salz Mehl, Pfeffer und Safran Veroneser Denar $44 \mathrm{~g} 8$ Ver. 6.

Auch in der zehnten Woche besorgte Hosser vom Beschließer Konrad 10 Rinder, 300 Käse, 3 1⁄2 Pazeiden Essig. In derselben vom Verwalter für Fische, Eier, Pfeffer und Safran, Schafe, Salz, Schmalz, Kräuter, Mehl Veroneser Denar 10 S 15 Ver. 4.

Auch in der elften Woche besorgte Hosser vom Beschließer Konrad bei der Familie Haslach 11 Rinder, 110 Käse. In derselben für Rinder- und Schafsfleisch, Käse, Kräuter, Pfeffer und Safran, Salz, Mehl, Schmalz und anderes zum Kochen Veroneser Denar 28 g 9 Ver. 10.

Auch in der zwölften Woche besorgte Hosser bei der Familie Haslach vom Beschließer Konrad 10 lebende Rinder, 300 Käse, 1 Maß Schmalz. In derselben vom Verwalter für 
Schafe, Eier, Mehl, Pfeffer und Safran, Salz und anderes für die Küche Veroneser Denar 29.

Auch in der dreizehnten Woche besorgte Hosser durch die Familie von Konrad dem Beschließer 10 Rinder, 250 Käse. In derselben vom Verwalter für 2 Rinder, Kräuter, Schafe, Pfeffer und Safran, Mehl, Schmalz und anderes für die Küche Veroneser Denar 18 wenigerg 2 .

Die Summe der Verwaltung m 25 lb 8 1/2, 30 Käse für S 40. Die Summe des BeschlieBer Konrad 81 Rinder, 72 Schafe, 32 1⁄2 Speckseiten ${ }^{53}$, 3585 Käse, 1 Maß Schmalz, 1 Ster Roggen, 22 Maß Bohnen, 7 Pazeiden Essig."

Mattia Pellegrini ist Lehramtsstudent im 7. Semester in den Fächern Geschichte, Sozialkunde und Politische Bildung und Geografie und Wirtschaftskunde.

Mattia.Pellegrini@uibk.ac.at

\section{Zitation dieses Beitrages}

Mattia Pellegrini, Ein Blick in Hossers Küche. Analyse der Rechnungslegung des Küchenmeisters Jakob Hosser über die bei der Belagerung der Burg Weineck (1292) aufgewendeten Lebensmittel, in: historia.scribere 13 (2021), S. 287-302, [http://historia. scribere.at], eingesehen 22.6.2021 (=aktuelles Datum).

(C) Creative Commons Licences 3.0 Österreich unter Wahrung der Urheberrechte der Autorlnnen. 